\title{
Logical Reasoning and Decision Making
}

\author{
David CC Ong, Souheil Khaddaj \\ Faculty of Computing, Information Systems and \\ Mathematics, \\ Kingston University, Kingston upon Thames,UK \\ \{K0018084, s.khaddaj\}@kingston.ac.uk
}

\author{
Rabih Bashroush \\ School of Computing, IT \& Engineering \\ University of East London \\ London, UK \\ Rabih@uel.ac.uk
}

\begin{abstract}
Most intelligent systems have some form of decision making mechanisms built into their organisations. These normally include a logical reasoning element into their design. This paper reviews and compares the different logical reasoning strategies, and tries to address the accuracy and precision of decision making by formulating a tolerance to imprecision view which can be used in conjunction with the various reasoning strategies.
\end{abstract}

Keywords-Intelligent Systems; Logical Reasoning; Decision Making

\section{Introduction}

Precision forms a basic fundamental precept of science, where every salient variable involved in a calculation must be taken into a consideration before a viable precise (or absolute) conclusion can be reached. But often this is not the case, since in many situations, particularly dynamic ones, there will always be some degree of inaccuracy / uncertainty in any conclusions reached from the actual findings. This uncertainty is the result of unconsidered variables, which have been intentionally or unintentionally excluded from the calculations or due to the imprecise elements in data analysis.

This dilemma has presented one of the greatest challenges for scholars, philosophers and scientists over the centuries. Immanuel Kant [1], Karl Popper [2] presented us with their views on this imprecision concept (e.g. definition of a truth) and Werner Heisenberg [3] gave us an explanation of the uncertainty principle. Lofti Zadeh [4] has presented a practical solution in the form of "fuzzy logic", which has led to the development of the soft computing based applications and approaches [5][6] in an attempt to offer a solution for coping with imprecise problems.

As well as the imprecision problems, this paper also tries to address the way basic learning processes may be achieved via a logical reasoning, by putting forward for a consideration Charles Peirce's [7] view of an additional classification of logical reasoning. It also, explores how logical reasoning process could be used to facilitate decision-making.

\section{Logical Reasoning Approaches}

Logical reasoning is an essential component in an intelligent system. It acts as a "brain" behind the decision-making process of the system [8]. Logical reasoning techniques rely heavily on the mathematical analysis of historical data (i.e. prior knowledge or experience), from simple probability calculation to more complex calculation such as the Bayesian method [9] and Nash equilibrium [10]. In fact, these techniques have been translated into wellestablished data mining methods for knowledge discovery, particularly in the field of neural networks [11]. The incorporation of these techniques has led to the formation of a self-learning capability in an intelligent system as it learns via a repetitive process of past experience [12].

However, the logical reasoning techniques of analysis, when coupled with a static configuration, have limited the ability of the system to react dynamically to changes in its environment. In an intelligent system, this static configuration is a core of the system's "self-belief" capability [13]. This core often requires a third-party (human) intervention to ensure the system is relevant to the current situation, since it has no ability itself to transform its core belief. The system should be "self-aware" of its environment, where a dynamic decision could be made to alter this core belief when there is a significant change observed in the environment.

Thus, a number of approaches have been developed to compensate for the inability of the some logical reasoning techniques to offer a viable solution without any human intervention, in the event of limited or non-existent historical data. Approaches using a heuristic strategy in the development of these techniques have been formulated [14]. Current heuristic techniques such as best-first search, LAO*, $\mathrm{A}^{*}$, etc. have been used particularly in the area of search algorithm and cost estimation, where a (fuzzy) pattern is used (shaped) to search for a viable solution in the decision-making process [15].

\subsection{Types of Logical Reasoning}

Deductive and Inductive Reasoning are widely adopted for basic logical analysis in modern science 
especially in investigative studies (e.g. prediction and statistical analysis). Deduction is the assumption derived from a set of facts, while induction is the generalisation of a set of facts. Charles Peirce has suggested abductive as an additional type of logical reasoning in which he has defined hypotheses (abduction) as a suggestion that something is possible [7].

We could further conclude that deduction is a rationalist (evidence) based approach, and it is completely certain. Induction is an empiricist (validation) approach where it is based on the outcome (conclusion) of an evaluation or experimentation and the outcome will be invalid when an additional premise is introduced that refutes the conclusion, thus it is a defensible approach. Abduction is a hypothesis based approach, where presumption is based on what is observed (or what is possible) without consideration of existing facts (or with very limited facts) which may be refuted with additional information [16][17]. These types of logical reasoning provide us with a means to distinguish received information and to react to it accordingly based on historical data.

\subsection{Logical Reasoning Characteristics}

\subsubsection{Deductive Reasoning}

Deductive reasoning provides a complete and certain solution, where typically there are two absolute types of responses, which are opposites (i.e. true or false). It is an introspective based approach, which governs the system's overall self-belief capability. It utilises the "imprinted directive" dataset to search for its responses. A set of instructions is stored in the "imprinted directive" dataset, where it describes how the system should work. Response time for this reasoning is considered fast as it does not perform any analysis calculation that may delay the response time. This kind of response could be identified as a basic intuition of the system.

\subsubsection{Inductive Reasoning}

Induction is based on the approximation and probability analysis of possible selections (generalisations) against the historical data by adopting a best selection strategy. An approximation (good enough) value and a list of possible values are provided because of this reasoning. It is based on the empirical (experiential) approach using metadata to search for its result(s). The outcome produced by this reasoning depends on time, as some of the older (or less frequent data) data might be removed from the metadata to ensure relevance of the process (experience) to the current situation. Response time for this reasoning may vary depending on the calculation (e.g. statistical analysis) conducted. When there is more than one solution found, abductive reasoning is consulted for selecting a possible solution.

\subsubsection{Abductive Reasoning}

Abductive reasoning is a heuristics approach which aims to provide possible solutions or alternatives, when other approaches have failed. This reasoning draws information from the "imprinted directive" data, the metadata and the historical data to form a "best educated-guess" for its responses (solutions). This heuristics approach can be represented by: -

a) random selection from the choices available from the metadata,

b) selection that may be close to or chosen by prior selections,

c) the successful conclusion of prior selections to form a selection, etc.

The nature of this heuristics approach enables this form of reasoning to respond instantaneously, but the accuracy of the response produced remains yet unknown. This characteristic is a form of selfinvestigative (exploration) capability, for while the accuracy of the response produced may remain in question, the level of accuracy could be determinate by revaluing reactions (consequence of the responses) received.

\subsection{Dataset of Logical Reasoning}

Data analysis is normally performed on a specific dataset. It is conducted by decision-making and learning processes, which are based on one or more types of logical reasoning approaches.

Deductive reasoning is conducted in situation where one set is a subset of another set (see Scenario A in figure 2) as the accuracy of the subset is assured. The subset in question remains true provided it evolves within its superset. As a result, a precise outcome of these facts can be produced.

Inductive reasoning is adopted when two sets of facts share commonalties in some part of their regions. Conflict or incompatible issues between these sets will arise from their uncommon regions (see Scenario B in figure 2). Thus, only generalisation of facts among these sets can be conducted to describe these facts as there will be some confliction of facts. As a result, various possible outcomes of these facts may be produced.

Abductive reasoning allows a boundary of the set to dynamically expand and contract; as it undergoes its own explorations (transformations) to unknown regions (see Scenario $C$ in figure 2). Exploration of unknown regions is identified as a self-investigatory ability. This self-investigatory ability is an essential component of an intelligent entity, which dictates and influences the self-belief mechanism in an intelligent entity. 


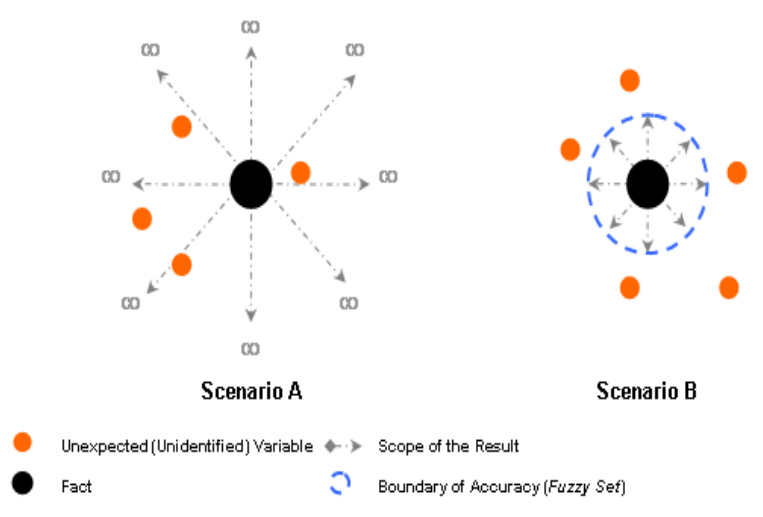

Figure 1 Accuracy of the theory scenarios

\section{Imprecision Problem}

Karl Popper urges that scientific theories should expose themselves in an attempt to follow observational and experimental refutation [2]. Inadvertently, he has suggested that a scientific theory without a boundary of accuracy would be useless owing to the inability to determine (or estimate) the level of inaccuracy that remains in the theory. A boundary of accuracy (truth) is established by falsification of a theory. As a result, the level of accuracy is verified given that the inaccuracy element of the theory has been identified and eliminated. Hence, the validity of a scientific theory is ensured within the established boundary of accuracy (i.e. acceptable values). Nevertheless, in dynamic situations, a boundary of accuracy may vary depending upon the environment wherein it resides since new unknown elements could surface which would perhaps in some way influence the theory. Thus, the establishment of a level of acceptable (or good enough) value could ensure the accuracy and acceptability of the theory itself.

Recognition of the level of an acceptable value as a form of fuzzy logic was first put forward to the scientific community in 1965 with the presentation by Lofti Zadeh of a paper, entitled "Fuzzy Set" [4], where he proposed the development of the fuzzy set theory. He offered an idea that a set of acceptable values, which approximates the actual value and are good enough to be used to represent that value.

Let us again reconsider Karl Popper's suggestions about a scientific theory without a boundary of accuracy as illustrated in figure 1 . Scenario A shows an abstract presentation of Karl Popper's assertions, where validity of the theory remains in question since every time the theory is applied, it has appeared to produce an inaccurate outcome as it has encountered unexpected variables. Identification of these unexpected variables helps to improve the overall level of accuracy of the theory. Thus, establishment of the boundary of accuracy (see Scenario B in figure 1) could ensure acceptance of the theory itself, where unexpected variables within the boundary have been identified and included as part of an acceptable outcome. This view is a form of fuzzy set, where it is a representative collection of acceptable (good enough) results produced by the theory or fact.

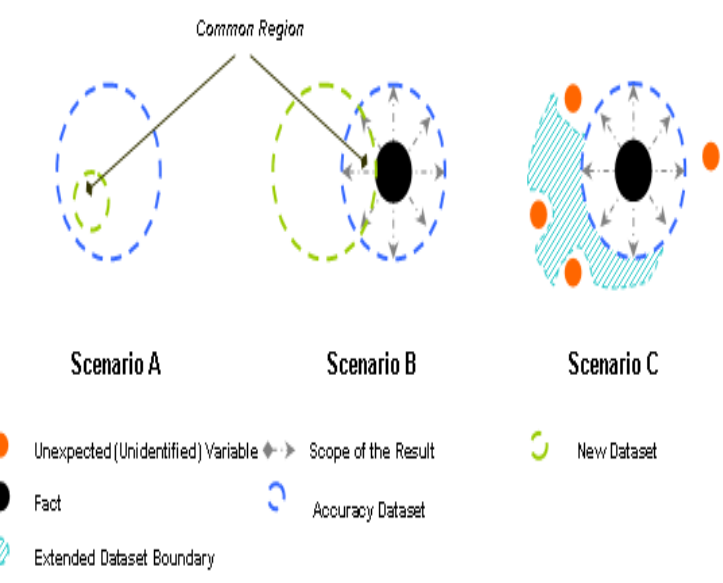

Figure 2 Dataset scenarios for logical reasoning

\section{Tolerance to Imprecision}

The theoretical concepts discussed earlier present us with the abstract aspects of logical reasoning in a dynamic learning process. The purpose of logical reasoning could not be achieved without a basis where comparison, assessment or judgement can be conducted, since information flow into the dynamic learning process might be rendered as meaningless. The classification and translation of this information into patterns will form a foundation for logical reasoning to conduct its operation. Therefore, the identification of a recognisable pattern helps to facilitate definition of the principle mechanism behind the decision-making process

\subsection{Pattern Template}

A pattern can emerge from a collection of facts, where some parts of these facts have shared common variables (e.g. objectives, features or values). Patterns can be recognised via a predefined basic characteristic of a pattern, potentially in the form of a template which is vital as a base for comparison. In other words, a pattern template provides a means to identify and evaluate a pattern from a collection of facts. This collection of facts is a form of fuzzy set. A proportion or absolute statement of the fact itself can represent the formation of this set. Establishment and recognition of a fuzzy set as a pattern facilitates the comparison process. 
A predefined pattern is used for a basis of comparison for a data analysis process [18][19]. Subjective perception plays a crucial role in determination of fuzzy set's classification. This perception can be classified as a predefined pattern's template.

\subsection{Pattern Assessment}

The ability to compare various patterns is a crucial part of decision-making process. The comparison is based on pattern template and observed pattern using identified measurable factor to form a conclusion (figure 3). Difference level of responses can be formulated based on this conclusion. This comparison is based on the principle behind the fuzzy set, where they are crosschecked to determine whether what has been observed corresponds with what is intended. Hence, observed patterns of behaviours could be monitored and managed based on the comparison of differences and commonalities of the patterns.

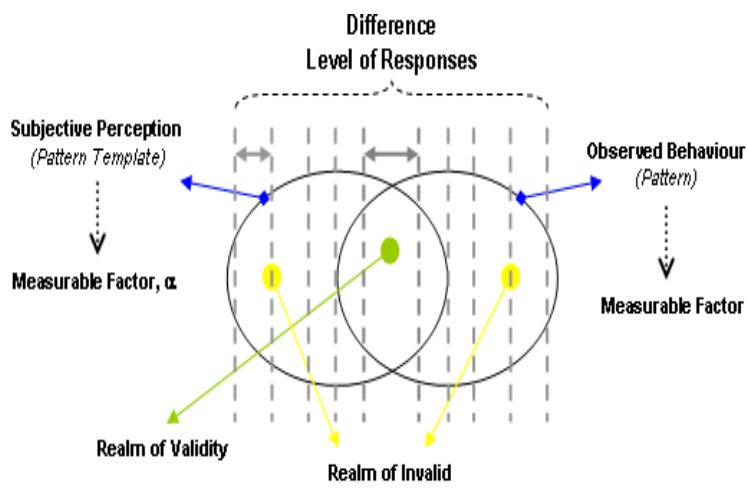

Figure 3 Illustration of pattern assessment

\subsection{Tolerance to Imprecision View}

Both of the conceptual theories proposed by Karl Popper and Lofti Zadeh could be combined to form a "Tolerance to Imprecision" view. This is defined by converting Karl Popper's theory on the accuracy of a scientific theory and subjective perception into Lofti Zadeh's fuzzy set.

This view asserts that a scientific theory could produce an infinite possible outcome depending on the ways it is applied, presented or viewed, thus the theory by default must have one universal fuzzy set, which consists of an "infinite result". When a theory is applied to a situation, a "valid result" can be produced, but when it is applied to different situations, a variation in the result may be created. In the dynamic situation, the possibility of obtaining an absolute result from a theory is viewed as unobtainable since there will be a slight variation in the result observed. Thus, a "boundary of accuracy" can be established by creating a "realm of acceptable results" in the form of a fuzzy set. This "acceptable results" set would be the subset of the "infinite result" set of the theory (figure 4).

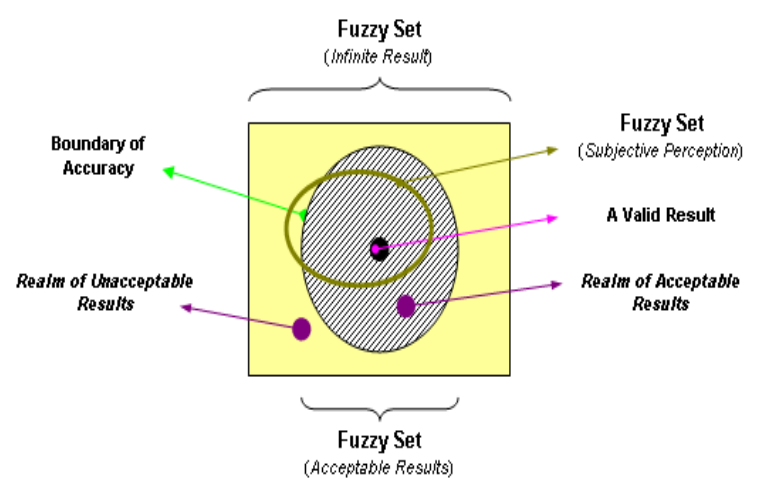

Figure 4 Illustration of "Tolerance to Imprecision"

By the establishment of these fuzzy sets, the validity of the theory can be ensured when it operates in an unknown novel and / or unsupervised situation. In addition, a subjective perception (or belief) of an individual could also influence the level of accuracy of a theory where it is perceived as a fuzzy set. This realm of "belief" resides in the "infinite result" set but it may be part of or dependent upon the acceptable results set. Thus, a "Tolerance to Imprecision" view suggests the following points: -

a) A scientific theory can produce an infinite possible outcome, which can be categorised into one fuzzy set.

b) It is unobtainable to achieve an absolute result that applies to every situation. Thus, a collection of acceptable results is identified to ensure the validity of the theory. This collection is a form of fuzzy set, which is a subset of the infinite result fuzzy set.

c) Subjective perception is form of fuzzy set, which is a subset of the infinite result fuzzy set but could be (or not be) part of the acceptable results fuzzy set.

d) The described fuzzy set is a form of pattern, where a subjective perception could be translated into a predefined template of pattern.

\section{Dynamic Decision-Making Framework}

Daily interaction in our lives consists of observation, analysis, formulation, reaction and forecasting, which provides us with the abilities to acknowledge or be aware of occurrences around us. Logical reasoning is a core substance behind these abilities as it facilitates and influences interaction of these abilities. Moreover, logical reasoning is an essential part of the learning process. This reasoning enables a learning process to form an "experience" capability, which could influence the whole decisionmaking process. 


\subsection{Dataset}

There are three dynamic datasets that can be used for data analysis in decision-making and learning processes. These are: -

a) "Historical" Dataset - any kind of transaction taking place in the decision-making process will be recorded (logged) in the historical database via a logging process.

b) "Metadata" Dataset - an experience in general terms can be described as a process of gathering knowledge by observation, responses and reactions to these responses for a given stimulus. Information derived from this metadata is presented as experience or knowledge of the system, which will thus have learned over a period of time

c) "Imprinted Directive" Dataset - the "imprinted directive" data holds a set of instructions describing how the system should behave. This "imprinted directive" dataset is a form of the system's basic intuition

\subsection{Learning Process}

The primary function of learning process is to perform various data analysis that could be translated into system's knowledge and experience, as well as to facilitate decision making components in revising the scope of the dataset allocated to them. A data analysis using inductive reasoning is performed on "historical" dataset to form system's experience. The produced results are then used to revise the scope of the "metadata" dataset that is exposed to the inductive decision maker. Data analysis uses Standard Deviation to obtain z-test value for transaction data stored in the "historical" dataset. This data analysis process support the self-learning ability of the system.

The data analysis using deductive reasoning is performed on "historical" dataset to form system's intuition. The produced results are then used to revise the scope of the "imprinted directive" dataset that is exposed to the deductive decision maker. Data analysis uses Standard Deviation to obtain very significant value for each type of transaction data. The significant value is identified as a high frequency for a particular standard deviation population (i.e. sigma value which is very close to 0 ). If a significant value with positive feedback is obtained, a point is added to a "significant score" in the "imprinted directive" dataset which is identified by the request and result type. On another hand, if a significant value with negative feedback is obtained, a point is reduced from the "significant score" in the "imprinted directive" dataset which is identified by the request and result type.

\subsection{Logging Process}

A logging process records every transaction occurring in the decision-making process in the "historical" dataset.

\subsection{Decision-Making Process Flow}

Deductive, inductive and abductive reasoning form an integral part of the dynamic learning and reasoning process flow (figure 5). Each type of logical reasoning has its own unique characteristics. For example, deductive reasoning may require a longer response period, while abductive reasoning can respond instantaneously.

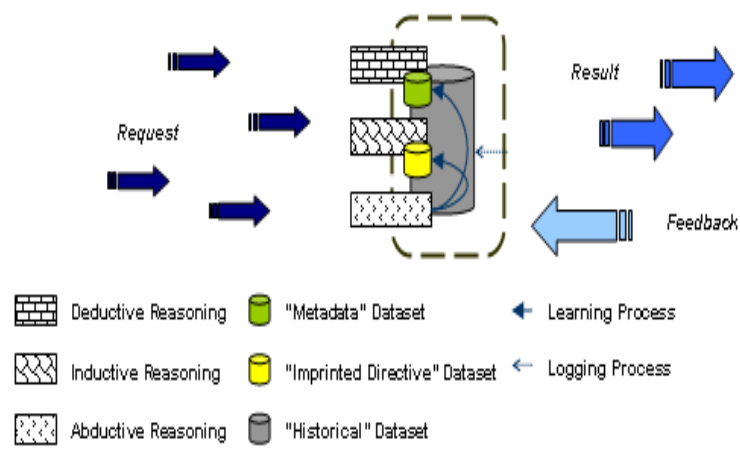

Figure 5 Interaction of decision-making processes

Capabilities of these types of logical reasoning have their own drawbacks, as well as their advantages, but when they are integrated as a single intelligent unit, the capabilities of each of these types of logical reasoning can compensate for each other's drawbacks in order to try achieving a better reasoning outcome. Based on actual circumstances in which time plays an important role, different reasoning strategies can be activated to produce a result. Therefore a best effort result is returned according to the system operating environment. For clarification of characteristics possessed by these types of logical reasoning, see table 1 .

\begin{tabular}{|c|c|c|c|}
\hline & \multicolumn{3}{|c|}{ Logical Reasoning } \\
\hline & Inductive & Deductive & Abductive \\
\hline Response period & Variable & Quick & Instant \\
\hline $\begin{array}{l}\text { Accuracy (from a } \\
\text { system point of view) }\end{array}$ & High & Absolute & Unknown \\
\hline $\begin{array}{l}\text { Ability to provide } \\
\text { alternative solutions }\end{array}$ & Possible & No & Yes \\
\hline Preferred Outcome & $\begin{array}{l}\text { Most desirable because it } \\
\text { has taken every factor } \\
\text { into consideration }\end{array}$ & $\begin{array}{l}\text { Desirable because it } \\
\text { trustits oun judgement } \\
\text { andlike lihood of } \\
\text { producing wrong } \\
\text { outome is low }\end{array}$ & $\begin{array}{l}\text { Least de sirable because } \\
\text { likelihood of wrong } \\
\text { outcome is very high }\end{array}$ \\
\hline $\begin{array}{l}\text { Core decision-making } \\
\text { feature }\end{array}$ & $\begin{array}{l}\text { Empinical Approach } \\
\text { (Conclusion of Facts) }\end{array}$ & $\begin{array}{c}\text { Intropective Approach } \\
\text { (Self-belief System) }\end{array}$ & $\begin{array}{l}\text { Heuristics Approach } \\
\text { (Educated Guess) }\end{array}$ \\
\hline
\end{tabular}

Table 1 Characteristics of logical reasoning 
However, the purpose of decision-making could not be achieved without a basis where comparison, assessment or judgement can be conducted, since data flow into a decision-making process might be rendered as meaningless. Classification of data as pattern is essential to form a basis for decisionmaking process to conduct its operation. A basic definition of pattern was presented in an attempt to help its identification and nature. Also, subjective perceptual characterization is offered to assist in the determination of the level of accuracy of the identified pattern in the form of a pattern template. In addition, the basic principle of comparison and assessment is defined by the tolerance to imprecision views (section 4.3).

\section{Conclusion}

In the quest to define an intelligent system, this paper started with the presentation of conceptual views of imprecision and logical reasoning notions. These notions have led to the creation of a "Tolerance to Imprecision" view with a presentation on how a pattern could be defined, identified and distinguished.

This paper also presented an integrated approach which involves a number of reasoning strategies and their comparison. Each strategy has its own advantages and disadvantages. For example, abductive reasoning tends to return quickest result but the quality of the result is questionable. On other hand, deductive reasoning returns the most accurate result as it performs empirical data analysis but this is expected to take longer time than abductive reasoning. It is proposed that combining reasoning strategies under a decision-making framework, decision production could continue within any circumstances, where time is a factor.

Moreover, when fully implemented, the proposed framework should be able to manipulate available resources intelligently to provide the best possible outcome (decision) relevant to its situation. In fact there are a number of potential computing fields that could benefit from the proposed framework such as 1) Supporting (assistant) Tool - assistant in a decision-making process; 2) Analytical, Forecasting and Predicting Model; 3) Data Mining (e.g. generating a summation analysis or identification of a new pattern in bulk information analysis); 4) Simulation \& Modelling - replication of an actual system in a limited controlled environment; 5) Best Effort System - a system that produces a best decision based on its scenario / environment, where the decision made may not fully realise all our expectations, and non-critical system, where further verification of the produced decision is conducted at a higher level of the system; 6) Search Engines (e.g. provides a list of possible outcomes based on the search keywords); and 7) Fault Tolerance Mechanisms. However, the evaluation of the proposed approach, including performance and reliability, initially using a networking load balancer will be carried out in future work.

\section{References}

[1] Abela, P, Kant's Empirical Realism (Oxford Philosophical Monographs) Clarendon Press, 2002.

[2] Popper, K. The Logic of Scientific Discovery. Page 1718. Routledge, 2006.

[3] Cassidy, Uncertainty: Life and Science of Werner Heisenberg. W.H. Freeman \& Co Ltd, 1994

[4] Zadeh, L, "Fuzzy Set", Information and Control, Vol 8, Academic Press, Inc., 1965, pp 338-353.

[5] Zadeh,, L, "Toward a generalized theory of uncertainty-An Outline", Information Sciences, 2005, pp. $1-40$.

[6] Zadeh, L "Toward a perception-based theory of probabilistic reasoning with imprecise probabilities", Journal of Statistical Planning and Inference, Elsevier Science, 2002 pp 233-264..

[7] Peirce, C (1993). Writings of Charles S. Peirce: A Chronological Edition: 1884-1886. Indiana University Press, 1993.

[8] Manna, Z, "Fundamentals of deductive program synthesis", IEEE Transactions on Software Engineering, 1992, pp. 674-704.

[9] Cheng, J, “AIS-BN: An adaptive importance sampling algorithm for evidential reasoning in large Bayesian networks", Journal of Artificial Intelligence Research, 2000, pp. 155-188.

[10] Gao, Y., "Meta-game Equilibrium for Multi-agent Reinforcement Learning". Advances in Artificial Intelligence, Springer, 2004, pp. 930-936.

[11] Guo, Y, "A Multiagent Approach for Logistics Performance Prediction Using Historical and Context Information", AAMAS'04, 2004, pp. 1164-1171.

[12] Ormoneit, D, "Kernel-based reinforcement learning in average-cost problem"s, IEEE Transactions On Automatic Control, 2002. pp. 1624-1636.

[13] Dagum, P, "Approximating Probabilistic Inference in Bayesian Belief Networks", IEEE Transactions on Pattern Analysis and Machine Intelligence, 1993, pp. 246-255.

[14] Cimatti, A, "Conformant planning via symbolic model checking and heuristic search", Artificial Intelligence, 2004, pp. 127-206.

[15] Hansen, E, "LAO*: a heuristic search algorithm", Artificial Intelligence, 2001, pp. 35-62.

[16] Paavola, S, "Abduction Through Grammar, Critic, And Methodeuti", Transactions of the Charles S. Peirce Society: A Quarterly Journal in American Philosophy, 2004, pp. 245-270.

[17] Bonet, B, "Planning with incomplete information as heuristic search in belief space”, AIPS2000, 2000.

[18] Uludag, U, "Biometric template selection and update: a case study in fingerprints", AVBPA, 2003, pp. 35-342.

[19] Guo, X, "A Stock Pattern Recognition Algorithm Based on Neural Networks",ICNC 2007. pp. 518-522. 